\section{The clinical value of in vivo confocal microscopy for diagnosis of ocular surface squamous neoplasia}

Y Xu', Z Zhou', Y Xu², M Wang'1, F Liu', H Qu' and J Hong ${ }^{1}$
OSSN before surgical resection. Although in vivo confocal microscopy cannot replace excisional biopsy for definitive diagnosis, it can be valuable for initial diagnosis and management of patients with OSSN.

Eye (2012) 26, 781-787; doi:10.1038/eye.2012.15; published online 9 March 2012

Keywords: in vivo confocal microscopy; diagnosis; ocular surface squamous neoplasia

\section{Introduction}

Ocular surface squamous neoplasia (OSSN) is the most common ocular surface tumor in adults and varying in prevalence from 0.02 to 3.5 cases per 100000 people. ${ }^{1}$ Risk factors may include ultraviolet light exposure, human papillomavirus infection, and possibly human immunodeficiency virus infection. The OSSN encompasses the diagnoses of conjunctival intraepithelial neoplasia (CIN), carcinoma in situ, and invasive ocular surface squamous carcinoma (OSSC). Clinically, OSSN most commonly arises in the interpalpebral limbus. The hyperplasia can be nodular, superficial, or diffusely invasive; it may present as localized slowly growing, mimic benign conjunctival degeneration or coexist with pinguecula and pterygium. Rarely, diffused tumors in elderly patients may be misdiagnosed as chronic unilateral conjunctivitis. Invasive squamous carcinoma can invade eyelid, orbit, adjacent paranasal sinus structures, and brain. However, systemic metastases resulted from OSSC are rare. ${ }^{1-3}$

Early diagnosis and treatment of OSSN can reduce the risk of this locally aggressive disease and improve the prognosis of patients by helping with localization of the tumor and
${ }^{1}$ Department of Ophthalmology, Peking University Eye Center, Peking University Third Hospital, Beijing, China

${ }^{2}$ Clinical Stem Cell Research Center, Peking University Third Hospital, Beijing, China

Correspondence: J Hong, Department of Ophthalmology, Peking University Eye Center, Peking University Third Hospital, No. 49 North Garden Road, Haidian District, Beijing 100191, China

Tel: + 861082266566 ;

Fax: + 861082089591

E-mail: hongjing1964@

sina.com

Received: 17 May 2011 Accepted in revised form: 23 December 2011 Published online: 9 March 2012 
preservation of vision. Recurrence rates are generally higher in more severe grades of OSSC and also depend on the adequacy of surgical margins at initial excision. In clinical practice, squamous conjunctival and corneal tumors are evaluated by pathological analysis through cytological or histopathological evaluation of tissue for the diagnosis of OSSN and determination of the subspecial types. ${ }^{4,5}$ In addition, superficial cytologic analysis, such as in vivo confocal microscopy, has also been reported to be a simple, safe, and relatively noninvasive method to diagnose OSSN. ${ }^{6-8}$ Moreover, in vivo confocal microscopy examination can also be used to distinct subspecial types of OSSN in addition to histopathological evaluation from tissue biopsy. ${ }^{9,10}$

The aim of this study was to investigate the clinical value of in vivo confocal microscopy in the diagnosis of OSSN, determination of subspecial type, and its concordance with histopathological biopsy analysis.

\section{Materials and methods}

\section{General information}

This study was approved by the Committee for Protection of Human Subjects in Research at the Faculty of Medicine, Peking University Third Hospital. All procedures were performed in accordance with the provisions of the Declaration of Helsinki for research involving human subjects and all five patients had signed an informed consent form before participating in this study. The five patients were recruited from the Ocular Surface and Cornea Outpatient Clinic of the Department of Ophthalmology, Peking University Third Hospital. Retrospective chart review was performed for this study from February 2008 to September 2010. Diagnosis for each patient was assessed by both in vivo confocal microscopy and pathological sections.

\section{Systemic and ocular examinations}

The initial systemic examination of all patients did not reveal any other tumor or disease process. The diagnosis of OSSN and subspecial type of OSSN was made by a cornea and ocular surface specialist. The initial diagnosis of OSSN and its subspecial type was mainly based on symptoms of patients, examinations of slit-lamp microscopy, and in vivo confocal microscopy, as all patients rejected a diagnostic biopsy before surgery. Routine ophthalmic examinations consisted of bestcorrected visual acuity (BCVA), slit-lamp microscopy examination, anterior-segment optical coherence tomography (AS-OCT), and in vivo confocal microscopy. Particular attention was paid to regions of lid margins, tarsal, bulbar conjunctiva, and cornea during examination of slit-lamp microscopy. None of the five patients had history of atopy, allergic diseases, StevensJohnson syndrome, chemical, thermal or radiation injury, any other ocular or systemic disorders, or had undergone any ocular surgery. ${ }^{11,12}$

\section{AS-OCT}

AS-OCT device (Visante OCT, Anterior segment imaging, Zeiss, Oberkochen, Germany) with iris-anterior segment and cornea modules was used in this study. In order to eliminate the effect of eyelid shelter, scans in both horizontal and vertical directions were applied. Images with the highest quality were saved for further analysis of the tumors.

\section{In vivo confocal microscopy}

In vivo confocal microscopy examination (Rostock Cornea Module/HRT II, Heidelberg, Germany) was performed. One drop of transparent ophthalmic gel (Tear-gel; Novartis, Mississauga, Ontario, Canada) was placed on the tip of the objective lens before the focal plane of the microscopy was manually scanned through the full OSSN thickness. Digital confocal images were captured at $10 \mu \mathrm{m}$ depth increments and stored during the scanning. The confocal fields were captured in both central and peripheral regions of the neoplasia, and each image presented an area of $400 \times 400 \mu \mathrm{m}^{2}{ }^{7,13}$

\section{Histopathology (HEE stain)}

The specimens of OSSC lesions were fixed in acetic acid for $20 \mathrm{~min}$ at room temperature, embedded in paraffin, and then cut into $6.0-\mu \mathrm{m}$ thick sections with a microtome. The tumor sections were rinsed in Harris hematoxylin, ammonia water, and eosin for $5 \mathrm{~min}$ each at room temperature and then washed with distilled water. The nucleus and cytoplasm of cell were stained brown and pink, respectively. The stained sections were subsequently examined under light microscopy after drying for $1 \mathrm{~h} .^{14,15}$

\section{Surgical procedures}

Every patient had signed a written informed consent before surgery. The surgery was performed under local anesthesia. Deep lamellar dissection was performed with Hessburg-Barron suction trephine to fully remove the OSSN, and the remaining corneal stromal, limbal, and conjunctival recipient tissue were trimmed with micro scissors. The removed OSSN and lamellar stroma of cornea were sent for histopathological examinations. The 
donor lamellar graft was taken from the whole layer of cornea. To prepare donor tissue, the corneoscleral rims were mounted epithelial side down onto a punch block and a $0.25-\mathrm{mm}$ oversized donor button was punched out with a trephine. The punched donor graft was transferred to the recipient stroma and scleral bed and sutured with 10-0 monofilament nylon sutures. ${ }^{16}$

\section{Results}

\section{Clinical features}

The five recruited patients with primary unilateral eyes of OSSN consisted with four men and one woman, aged between 65 and 81 years (mean \pm SEM: $72.4 \pm 6.54$ years old). The average BCVA of them was 20/73 (Snellen) and all patients presented ambulatory clinical complains before surgery, including ocular redness, tearing, blurred vision, symptoms of eye fatigue, irritation, and foreign body sensation (Table 1). Slit-lamp examination revealed a red gelatinous tumor with vascular dilatations invading the temporal, superior, or inferior bulbar conjunctiva in all the patients. There was also peripheral corneal invasion of the tumors from 90 to $270^{\circ}$ of limbal circumference in all the patients (Figure 1).

\section{AS-OCT}

The AS-OCT examination showed one tumor in each patient. Tumors arose from limbal and invaded into conjunctival and corneal surface, where normal structures of conjunctival and corneal epithelium layer were destroyed and the density of large fibrovascular structures became irregular. The tumor did not invade the Bowman's layer in three patients (CIN, Figure 2a and b), invaded the Bowman's layer in one patient (carcinoma in situ, Figure 2c), and invaded beneath the Bowman's layer and into anterior stroma in another patient (OSSC, Figure 2d).

\section{In vivo confocal microscopy}

In vivo confocal microscopy of the OSSN at the epithelial layer revealed a number of abnormal squamous epithelial cells, which were enlarged, irregular, and pleomorphic in shape. Highly reflective squamous

Table 1 Clinical characteristics of patients before surgery

\begin{tabular}{llccll}
\hline Patient no. & Gender/age & History of lesion (months) & Size of lesion $(\mathrm{mm})$ & BCVA & Complications \\
\hline 1 & M/67 & 5.8 & $6.3 \times 7.8$ & $20 / 40$ & Tearing, irritation \\
2 & M/65 & 4.6 & $5.0 \times 6.2$ & $20 / 25$ & Tearing, conjunctival hyperemia \\
3 & M/73 & 5.4 & $6.5 \times 7.0$ & $20 / 50$ & Tearing, irritation \\
4 & F/81 & 6.3 & $5.8 \times 8.6$ & $20 / 100$ & Tearing, conjunctival hyperemia \\
5 & M/76 & 5.8 & $16.4 \times 12.9$ & $20 / 40$ & Tearing, irritation \\
\hline
\end{tabular}
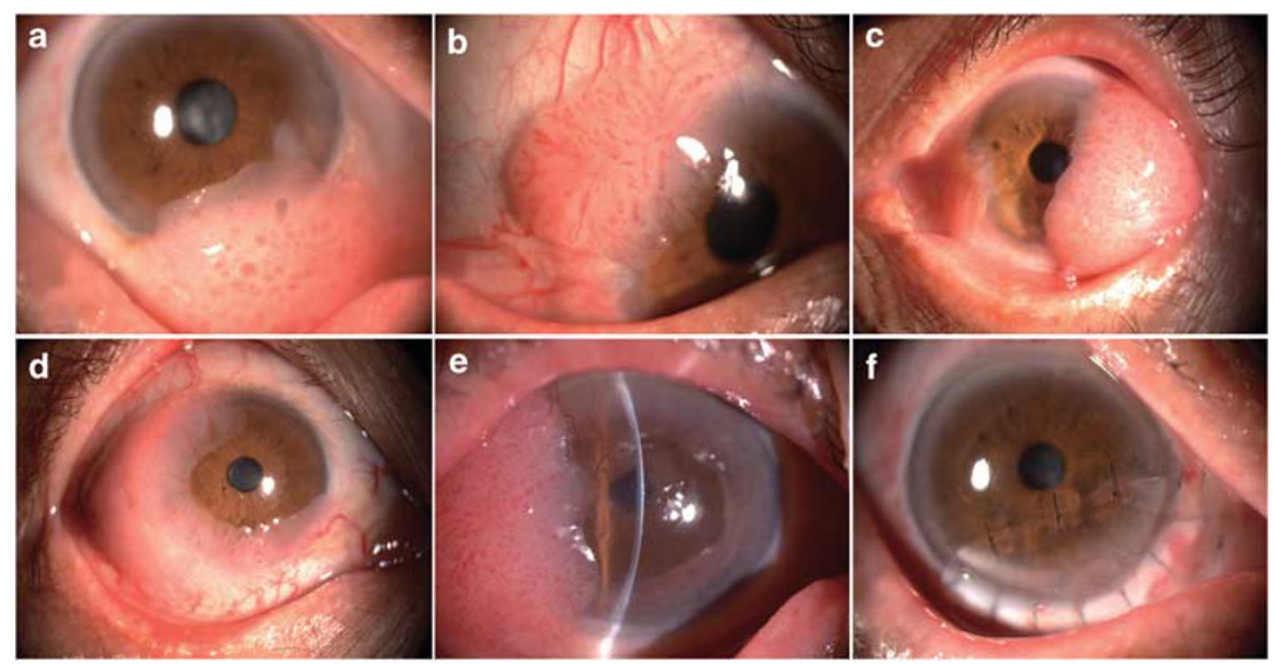

Figure 1 Anterior-segment photography of the tumors. The tumors revealed red gelatinous appearance with vascular dilatations invading the temporal, superior, or inferior bulbar conjunctiva in all the patients. The tumors invaded peripheral corneal limbal circumference for $90-180^{\circ}$ (CIN) (a-c), $240^{\circ}$ (carcinoma in situ) (d), and $270^{\circ}$ (OSSC) (e). (f) OSSN was excised and partial lameral keratoplasty was performed. 

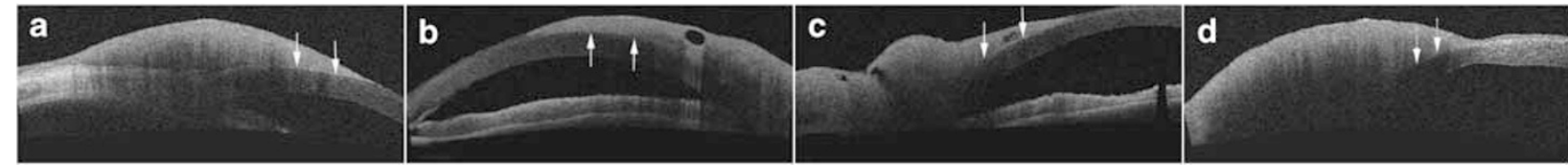

Figure 2 AS-OCT findings of the tumors. AS-OCT revealed that the extensive abnormal ocular surface tumors arose from limbal and invaded into conjunctival and corneal surface, where normal structures of conjunctival and corneal epithelium layer were destroyed and the density of large fibrovascular structures became irregular. $(a, b)$ The tumor did not invade the Bowman's layer in three patients diagnosed as CIN (white arrows). (c) The tumor invaded the Bowman's layer in one patient diagnosed as carcinoma in situ (white arrow with big head). (d) The tumor invaded beneath the Bowman's layer and into anterior stroma in another patient diagnosed as OSSC (white dashed arrows).
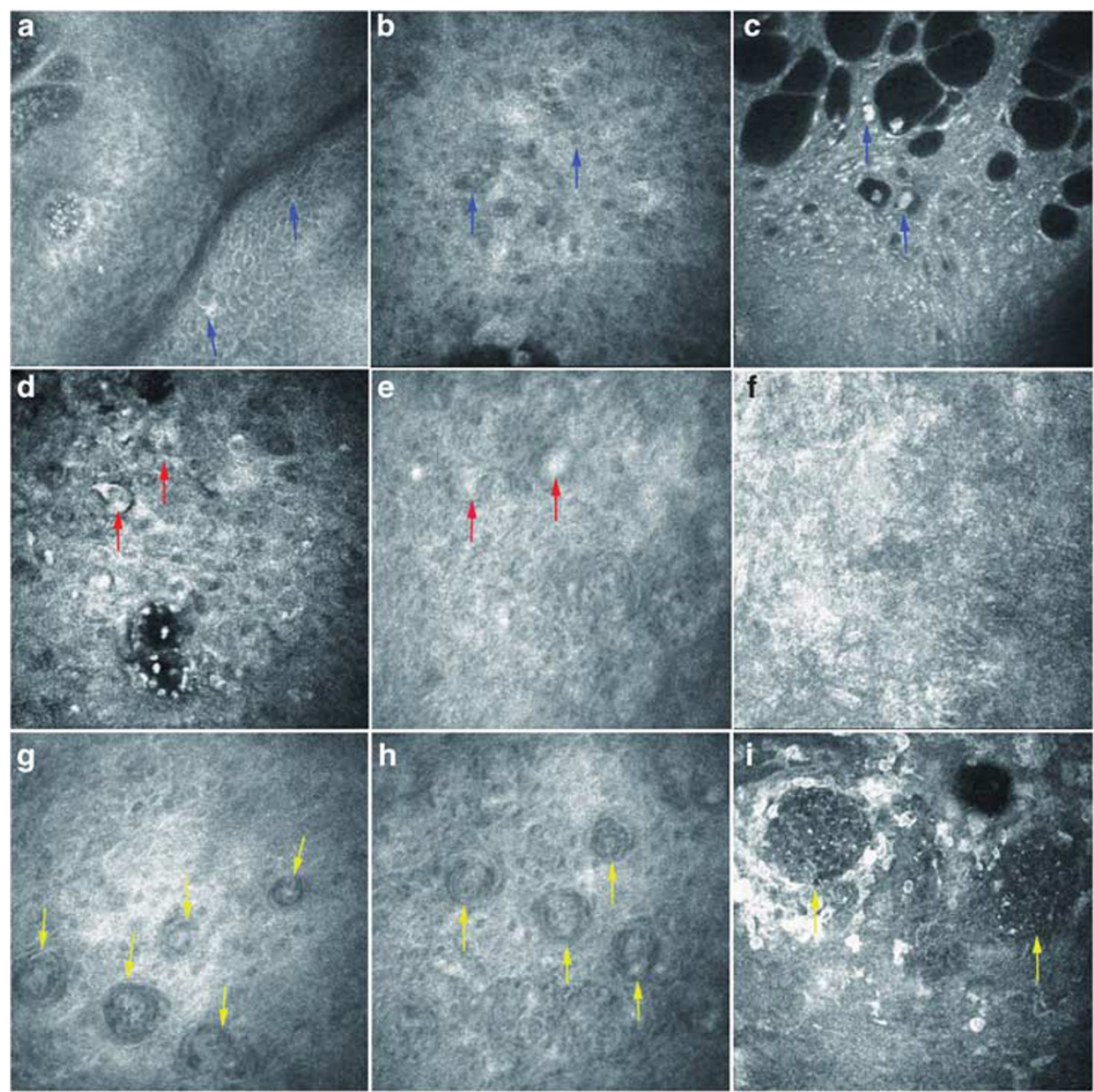

Figure 3 Representative pictures from in vivo confocal microscopy. In vivo confocal microscopy on the OSSN revealed different types of abnormal squamous epithelial cells. In three cases diagnosed as CIN, (a) abnormal squamous epithelial cells were enlarged, irregular, and pleomorphic in shape (blue arrows), (b) cellular anisocytosis and anisonucleosis (blue arrows), (c) enlarged nuclei with high nuclear to cytoplasmic ratio, highly reflective cytoplasm, and indistinct cytoplasmic borders (blue arrows) were observed. (d, e) Isolated, keratinized, binucleated, and actively mitotic dysmorphic epithelial cells were observed in one case diagnosed as carcinoma in situ (red arrows. (f) No invasive cells were observed in the stroma layer in patients with carcinoma in situ. (g, h) Nests were partially formed in one case diagnosed as OSSC (yellow arrows). (i) Squamous neoplastic cells invaded into basement membrane, the Bowman's layer, and anterior stroma layer in the same case to form nests (yellow arrows).

epithelial cells, cellular anisocytosis and anisonucleosis, enlarged nuclei with high nuclear to cytoplasmic ratio, high reflective cytoplasm, and indistinct cytoplasmic borders were observed in three cases diagnosed to be CIN (Figure 3a-c). Isolated, keratinized, binucleated, and actively mitotic dysmorphic epithelial cells featured carcinoma cells in the other two cases (Figure $3 \mathrm{~d}-\mathrm{f}$ ), in which nests were partially formed in one case and squamous neoplastic cells extended below basement membrane into the Bowman's layer and anterior stromal 
layer in the other case (Figure $3 g-i)$. The latter two cases were diagnosed to be carcinoma in situ and OSSC, respectively.

\section{Histopathological analysis (HEE stain)}

Histopathological cytomorphologic study of excised OSSN was assessed postoperatively in all the five patients. Among the four cases with abnormal squamous epithelial cells, which were enlarged, irregular, and pleomorphic in cellular shape, anisocytosis, and anisonucleosis, three of those were diagnosed to be CIN (Figure $4 \mathrm{a}$ and $\mathrm{d}$ ), whereas the other one case was suspected to be carcinoma in situ (Figure $4 \mathrm{~b}$ and e). The other one case was diagnosed to be invasive OSSC, which showed isolated, keratinized, binucleated, and actively mitotic dysmorphic epithelial cells with enlarged nuclei, high nuclear to cytoplasmic ratio, indistinct cytoplasmic borders, and partially formed nests (Figure 4c and f). Both the morphological features and the invasive extents of the tumors were concordant between in vivo confocal microscopy findings and the histopathological analysis.

\section{Discussion}

Ocular surface cytological examination with in vivo confocal microscopy is a simple, safe, and relatively noninvasive diagnostic tool that has been underused in the diagnosis and follow-up caring of patients with OSSN. ${ }^{17-19}$ From our experience, in vivo confocal microscopy could help with the initial clinical diagnosis of OSSN, including subspecial types, estimation of recurrence, management of treatment, and evaluation of response to topical chemotherapeutic agents in patients with conjunctival and corneal squamous lesions. When compared with a subsequent biopsy, cytological evaluation with in vivo confocal microscopy was capable to distinguish different stages of OSSN in all the cases.

These findings were also supported by other studies comparing the images from in vivo confocal microscopy with cytological samples obtained by either scraping or histopathological biopsy. ${ }^{20,21}$

Recent studies suggest that the accuracy of diagnosis with cytological in vivo confocal microscopy could be improved by the implementation of automated image analysis. This technique has been widely used in the interpretation of conjunctival and corneal exfoliative cytological analysis, and the acquired characteristics of cells were similar to those obtained from biopsies of OSSN. ${ }^{22}$ Ocular surface cytological examinations, such as in vivo confocal microscopy, have several advantages in the diagnosis and management of OSSN. It is minimally invasive, almost painless, and can be performed in regular office settings. These features are useful when treating debilitated patients or patients who refuse to experience a more invasive surgery. Moreover, cytological image analysis can be transferred immediately to pathologists or ophthalmologists, elevating the efficiency of diagnosis. In addition, in vivo confocal-microscopy sampling from various ocular surface regions can offer a real-time monitoring of the

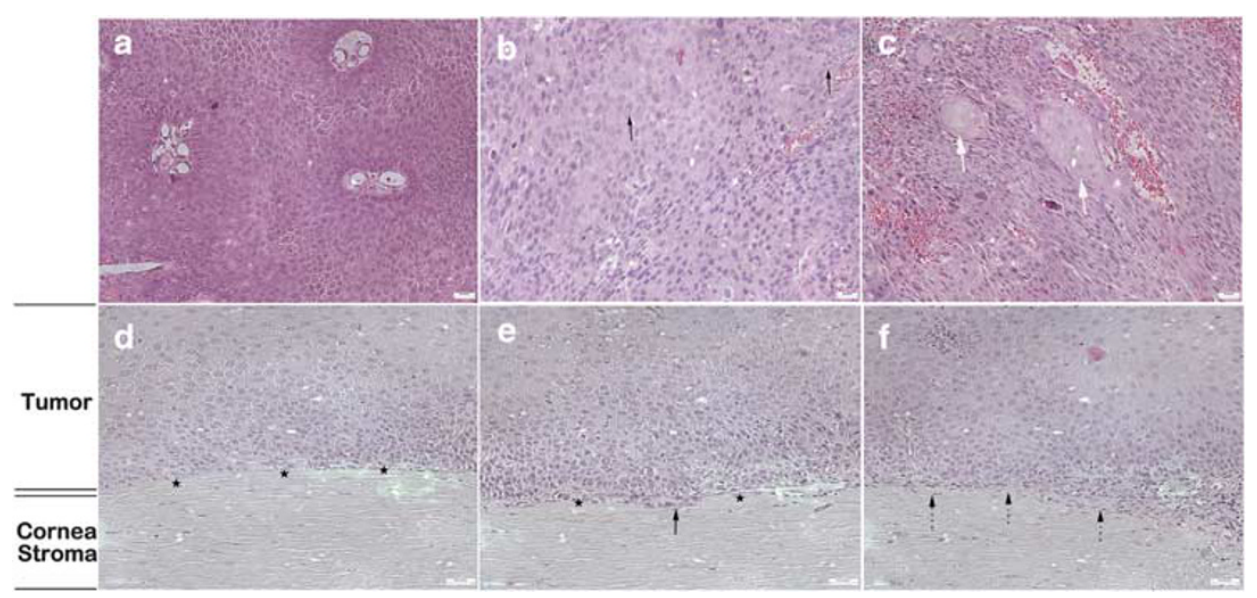

Figure 4 Histopathological analysis of OSSN by H\&E staining. (a) Enlarged and irregular squamous epithelial cells with abnormal density of cells (CIN). (b) Binucleated, actively mitotic dysmorphic cells (black arrows) and cells with increased nuclear/cytoplasm ratio were observed in patients diagnosed as carcinoma in situ. (c) Heteromorphic abnormal epithelial cells with partially formed carcinoma cell nests in OSSC were revealed (thick white arrows). The abnormal squamous epithelial cells (d) did not invade the Bowman's layer (black stars) in three cases diagnosed as CIN; (e) invaded but did not penetrate the Bowman's layer (black stars) in one case diagnosed as carcinoma in situ (black arrow); and (f) invaded beneath the Bowman's layer and into anterior stroma in the other case diagnosed as OSSC (black dashed arrows). 
extent and condition of tumor, and guide further managements. However, comparison of concordance between pathologists and in vivo confocal microscopy with automated image analysis still deserves further investigation. Moreover, ocular surface in vivo confocalmicroscopy examination has its limitations. For instance, the maximum examining depth of this technology is $500 \mu \mathrm{m}$, which impedes the detection of tumors inside the eye.

As demonstrated in this study, in vivo confocalmicroscopy findings and the histopathological analysis showed high concordance in both the morphological features and the invasive extents of the tumors, which will be discussed into details later. Briefly, in vivo confocal-microscopy analysis can be reliable in predicting the grade of dysplasia that based on cellular morphology, nuclear atypia, and nuclear to cytoplasmic ratio. The grading of OSSN also depends on the extent of the epithelial involvement that is better assessed by in vivo confocal microscopy compared with well-orient biopsy. In addition, cytological in vivo confocalmicroscopy assessment can differentiate between invasive and in situ tumors. A relatively accurate diagnosis and staging of carcinoma can be made from either histopathological evaluation of biopsies or cytological in vivo confocal-microscopy assessment, although both techniques occasionally give false positive or false negative diagnosis. ${ }^{23-25}$

In our study, in vivo confocal microscopy revealed different pictures of squamous neoplastic epithelial cells in different stages of OSSN. First, in three of the cases, abnormal squamous epithelial cells were enlarged, irregular, and pleomorphic in shape. Highly reflective cells, cellular anisocytosis and anisonucleosis, enlarged nuclei with high nuclear to cytoplasmic ratio, high reflective cytoplasm, and indistinct cytoplasmic borders were observed. In the meantime, consistent findings that enlarged and irregular squamous epithelial cells with abnormal density of cells were revealed by histopathological analysis in the same three cases to be diagnosed as CIN. Second, isolated, keratinized, binucleated, and actively mitotic dysmorphic epithelial cells were observed by in vivo confocal microscopy; in the other case, no invasive cells were observed in the stromal layer. In pathological sections from the same case, binucleated, actively mitotic dysmorphic, and increased nuclear/cytoplasm ratio of neoplastic cells were observed, which invaded the Bowman's layer without stroma involvement and derived a diagnosis of carcinoma in situ. Third, in the last case, in vivo confocal microscopy showed that nests were partially formed while squamous neoplastic cells invaded into basement membrane, the Bowman's layer, and anterior stromal layer, whereas identical findings were observed from the histopathological analysis that heteromorphic epithelial cells with partially formed carcinoma cell nests invaded beneath the Bowman's layer and into anterior stroma. Therefore, high consistence was showed between in vivo confocal-microscopy findings and the histopathological analysis in all the five cases from three different stages of OSSN.

Surgical excision of different subtypes of cornea and CIN, including carcinoma in situ and invasive OSSC, has been reported to be associated with different recurrence. The CIN, carcinoma in situ, has relatively low recurrent rate, whereas the invasive OSSC has a high rate of recurrence. One reason for the recurrence of tumor is that the edges and deep margins of tumor are generally difficult to determine. The tumor edges are commonly clear and avascular, leading to a false impression that the tumor looks smaller than it actually is. All the five cases recruited in this study showed large lesions with corneal involvement. Surgical excision of such lesions with safety margins could have left large defected ocular tissues that might require corneal graft. During surgical excision, total lesions were removed with minimal damage to the ocular surface. ${ }^{13,26,27}$

This study suggested that ocular surface cytological in vivo confocal-microscopy examination was helpful in initial evaluation of subspecial types of OSSN and could also be valuable in the setting of suspected recurrent tumors and in the follow-up evaluation of patients on topical chemotherapeutic agents. Although cytological in vivo confocal microscopy cannot replace biopsy, it can have an important role in the diagnosis and management of patients with OSSN in a noninvasive manner.

\section{Summary}

What was known before

- Early diagnosis and treatment of OSSN can decrease the risk of locally aggressive disease, improve the patient's prognosis for local control, and help with preservation of vision. In clinical practice, squamous conjunctival and corneal tumors are evaluated by pathological analysis through cytological or histopathological evaluation of tissue for the diagnosis of OSSN and subspecial types. In addition, superficial cytological analysis, such as in vivo confocal microscopy, has also been reported to be a simple, safe, and relatively noninvasive diagnostic tool to differentiate between CIN, carcinoma in situ, and invasive OSSC in the cell levels.

What this study adds

- The application of in vivo confocal microscopy in diagnosis of OSSN has to be first approved and then can be more widely used in clinics. Therefore, we investigated the clinical value of application of in vivo confocal microscopy in diagnosis of OSSN, determination of subspecial type, and its concordance with histopathological biopsy analysis. 


\section{Conflict of interest}

The authors declare no conflict of interest.

\section{References}

1 Lee GA, Hirst LW. Ocular surface squamous neoplasia. Surv Ophthalmol 1995; 39: 429-450.

2 Tunc M, Char D, Crawford B, Miller T. Intra-epithelial and invasive squamous cell carcinoma of the conjunctiva: analysis of 60 cases. Br J Ophthalmol 1999; 83: 98-103.

3 Finger PT, Tran H, Turbin RE, Perry HD, Abramson DH, Chin K. High-frequency ultra sonographic evaluation of conjunctival intra-epithelial neoplasia and squamous cell carcinoma. Arch Ophthalmol 2003; 121: 168-172.

4 Tulvatana W, Tirakunwichcha S. Multifocal squamous cell carcinoma of the conjunctiva with intra ocular penetration in a patient with AIDS. Cornea 2006; 25: 745-747.

5 Shields CL, Manchandia A, Subbiah R, Eagle Jr RC, Shields JA. Pigmented squamous cell carcinoma insitu of the conjunctiva in 5 cases. Ophthalmology 2008; 115: 1673-1678.

6 Vemuganti GK, Naik MN, Honovar SG, Sekhar GC. Rapid intraoperative diagnosis of tumors of the eye and orbit by squash and imprint cytology. Ophthalmology 2004; 111: 1009-1015.

7 Duchateau N, Hugol D, D'Hermies F, Meyer A, Labbé A, Dupas B et al. Contribution of in vivo confocal microscopy to limbal tumor evaluation. J Fr Ophtalmol 2005; 28: 810-816.

8 Espana EM, Grueterich M, Romano AC, Touhami A, Tseng SC. Idiopathic limbal stem cell deficiency. Ophthalmology 2002; 109: 2004-2010.

9 Tervo T, Moilanen J. In vivo confocal microscopy for evaluation of wound healing following corneal refractive surgery. Prog Retin Eye Res 2003; 22: 339-358.

10 Dawson DG, Edelhauser HF, Grossniklaus HE. Long-term histopatho- logic findings in human corneal wounds after refractive surgical procedures. Am J Ophthalmol 2005; 139: 168-178.

11 Cartsburg O, Kersten A, Sundmacher R, Nadjari B, Pomjanski N, Böcking A. Treatment of 9 squamous epithelial carcinoma in situ lesions of the conjunctiva (CIN) with mitomycin $C$ eye drops in cytological and DNA image cytometric control. Klin Monatsbl Augenheilkd 2001; 218: 429-434.

12 Karp CL, Moore JK, Rosa Jr RH. Treatment of conjunctival and corneal intraepithelial neoplasia with topical interferon a-2b. Ophthalmology 2001; 108: 1093-1098.

13 Malandrini A, Martone G, Traversi C, Caporossi A. In vivo confocal microscopy in a patient with recurrent conjunctival intraepithelial neoplasia. Acta Ophthalmol Scand 2008; 86 : 690-691.

14 Vann RR, Karp CL. Perilesional and topical interferon alfa-2b for conjunctival and corneal neoplasia. Ophthalmology 1999; 106: 91-97.

15 Frucht-Pery J, Rozenman Y, Pe'er J. Topical mitomycin-C for partially excised conjunctival squamous cell carcinoma. Ophthalmology 2002; 109: 548-552.

16 Shields C, Shields J. Tumors of the conjunctiva and cornea. Surv Ophthalmol 2004; 49: 3-24.

17 Balestrazzi A, Martone G, Pichierri P, Tosi GM, Caporossi A. Corneal invasion of ocular surface squamous neoplasia after clear corneal phacoemulsification: in vivo confocal microscopy analysis. J Cataract Refract Surg 2008; 34(6): 1038-1043.

18 Gentile CM, Burchakchi AI, Oscar CJ. In vivo confocal microscopy study of ocular surface neoplasia manifesting after radial keratotomy and laser in situ keratomileusis. Cornea 2009; 28(3): 357-359.

19 Dogru M, Katakami C, Miyashita M, Hida E, Uenishi M, Tetsumoto $\mathrm{K}$ et al. Visual and tear function improvement after superficial PTK for mid-stromal corneal scarring. Eye 2000; 14: 779-784.

20 McKelvie PA, Daniell M. Impression cytology following mitomycin-C therapy for ocular surface squamous metaplasia. Br J Ophthalmol 2001; 85: 1115-1119.

21 Parrozzani R, Lazzarini D, Dario A, Midena E. In vivo confocal microscopy of ocular surface squamous neoplasia. Eye 2011; 25(4): 455-460.

22 Hassani RT, Brasnu E, Amar N, Gheck L, Labbe A, Sterkers $\mathrm{M}$ et al. Contribution of in vivo confocal microscopy to diagnosis of invasive ocular surface squamous neoplasia: a case report. J Fr Ophtalmol 2010; 33(3): 163-168.

23 Nolan GR, Hirst LW, Bancroft BJ. The cytomorphology of ocular surface squamous neoplasia by using impression cytology. Cancer 2001; 93: 60-67.

24 Tseng SH, Chen YT, Cheng HC, Huang FC, Lee SC, Chen FK. Impression cytology study of conjunctival epithelial phenotypes on the healing ocular surface after pterygium excision. Cornea 2001; 20: 244-250.

25 Shelil AE, Shields CL, Shields JA, Eagle Jr RC. Aggressive conjunctival squamous cell carcinoma in a patient following liver transplantation. Arch Ophthalmol 2003; 121: 280-282.

26 Balestrazzi A, Martone G, Pichierri M, Tosi GM, Caporossi A. Corneal invasion of ocular surface squamous neoplasia after clear corneal phacoemulsification: in vivo confocal microscopy analysis. J Cataract Refract Surg 2008; 34: 1038-1043.

27 Giaconi JA, Karp CL. Current treatment options for conjunctival and corneal intra-epithelial neoplasia. Ocul Surf 2003; 1: 66-73. 